\title{
AVALIAÇÃO DE TIPOS DE ONDAS GERADAS POR DOIS MODELOS DE TRANSDUTORES PARA DETERMINAÇÃO DO MÓDULO DE ELASTICIDADE DINÂMICO
}

\author{
Diego Martins Stangerlin ${ }^{1}$, Joaquim Carlos Gonçalez ${ }^{2}$, Raquel Gonçalves ${ }^{3}$, Elio José Santini ${ }^{4}$, \\ Leandro Calegari ${ }^{5}$, Rafael Rodolfo de Melo ${ }^{6}$, Darci Alberto Gatto ${ }^{7}$ \\ ${ }^{1}$ Eng. Florestal, M.Sc., Instituto de Ciências Agrárias e Ambientais, UFMT, Sinop, MT, Brasil - stangerlin@ufmt.br \\ ${ }^{2}$ Eng. Florestal, Dr., Depto. de Ciências Florestais, UnB, Brasília, DF, Brasil - goncalez@unb.com.br \\ ${ }^{3} \mathrm{Eng}^{\mathrm{a}}$ Civil, Dr ${ }^{\mathrm{a}}$., Depto. de Construções Rurais, UNICAMP, Campinas, SP, Brasil - raquel@ feagri.unicamp.br \\ ${ }^{4}$ Eng. Florestal, Dr., Depto. de Ciências Florestais, UFSM, Santa Maria, RS, Brasil - ejsantini@ gmail.com \\ ${ }^{5}$ Eng. Florestal, Dr., Centro de Saúde e Tecnologia Rural, UFPB, Patos, PB, Brasil - leandrocalegari@ yahoo.com.br \\ ${ }^{6}$ Eng. Florestal, M.Sc., Campus Universitário Cinobelina Elvas, UFPI, Bom Jesus, PI, Brasil - rrmelo2@ yahoo.com.br \\ ${ }^{7}$ Eng. Florestal, Dr., Depto. de Engenharia Agrícola, UFPel, Pelotas, RS, Brasil - darcigatto@ yahoo.com
}

Recebido para publicação: 14/07/2009 - Aceito para publicação: 06/12/2009

\begin{abstract}
Resumo
O objetivo deste estudo foi de avaliar os tipos de ondas gerados por dois modelos de transdutores utilizados para determinação do módulo de elasticidade dinâmico por meio de ensaios com ultrassom e correlacioná-los ao módulo de elasticidade estático obtido por compressão paralela às fibras. Para tanto, utilizou-se um aparelho de ultrassom dotado de transdutores faces planas e de pontos secos com freqüência de $50 \mathrm{kHz}$. A velocidade ultra-sônica foi determinada ao considerar a transmissão da onda ao longo do comprimento de amostras de madeira com dimensões nominais de 5 x 5 x $20 \mathrm{~cm}$. Para avaliar a sensibilidade do método ultra-sonoro e os tipos de ondas gerados pelos respectivos transdutores, as amostras foram ensaiadas destrutivamente à compressão paralela, com determinação do módulo de elasticidade por ambas metodologias. Apesar da diferença de valores absolutos do módulo de elasticidade entre os ensaios de ultrassom com transdutores faces planas, que geraram ondas longitudinais, e os ensaios de compressão paralela, em função da natureza viscoelástica da madeira, verificou-se uma boa correlação entre os dados. Com relação ao uso dos transdutores pontos secos, que geraram ondas de superfície, não foi verificada boa correlação com os ensaios destrutivos.

Palavras-chave: Ondas longitudinais; ondas de superfície; transdutores de faces planas; transdutores de pontos secos; ensaios não-destrutivos.
\end{abstract}

\begin{abstract}
Evaluation of wavess generated for two models of transducers for determining the dynamic modulus of elasticity. This study aimed to evaluate the types of waves generated for two transducers used for determining the dynamic modulus of elasticity by tests with ultrasound and to correlate them to the static modulus of elasticity obtained by parallel compression. For this study ultrasonic equipment with planes faces and point-contact transducers of $50 \mathrm{kHz}$ was used. The ultrasonic speed was determined considering the transmission of the wave along the length of the samples with nominal dimensions of $5 \times 5 \times 20 \mathrm{~cm}$. To evaluate the sensitivity of the ultrasonic method and the types of waves generated for the transducers, samples were submitted to destructive conventional parallel compression, with determination of the elasticity modulus for both methodologies. Although the difference of absolute values between tests of ultrasound with transducers planes faces, that generated longitudinal waves, and parallel compression, due to wood viscoelastic nature, there was a good correlation between the obtained data. It was not observed good correlation between the use of point-contact transducers, which generated surface waves, and the destructive tests.

Keywords: Longitudinal waves; surface waves; planes faces transducers; point-contact transducers; not-destructive methods.
\end{abstract}

\section{INTRODUÇÃO}

Embora a hipótese básica para avaliação não-destrutiva da madeira tenha sido proposta por Jayne em 1959, no Brasil essa técnica começou a ser estudada apenas no final da década de 1980. Seu 
idealizador afirma que a armazenagem de energia e as propriedades de dissipação da madeira, que podem ser medidas por meio não-destrutivo, são controladas pelos mecanismos que determinam o comportamento desse material frente a solicitações estáticas. Microscopicamente, as propriedades de armazenamento de energia são controladas pela orientação das células e por sua composição estrutural, aspectos que influenciam as características elásticas da madeira. Essas propriedades são frequência de oscilação na vibração ou transmissão da velocidade de propagação da onda. Desse modo, as medidas das taxas de deterioração de vibrações livres ou atenuação de ondas acústicas são utilizadas para se observar a propriedade de dissipação de energia na madeira.

Segundo Wang et al. (2007), significativos esforços vêm sendo direcionados para o desenvolvimento de uma tecnologia consistente de avaliação não-destrutiva capaz de predizer com eficácia as propriedades intrínsecas da madeira.

Com base nos princípios da propagação de ondas mecânicas, a avaliação não-destrutiva de madeiras por meio da técnica de emissão de ondas de ultrassom, atualmente, aparece como sendo um dos métodos mais aplicados e promissores, em função da facilidade de operação e custo relativamente baixo na aquisição e operacionalização do equipamento, quando comparado aos equipamentos utilizados em ensaios destrutivos.

De acordo com Gonçalez et al. (2001), classificam-se como ondas de ultrassom as ondas acústicas de frequência superior a $20 \mathrm{kHz}$. O método ultrassonoro se baseia na análise da propagação e sua relação entre a resposta ao ultrassom e às constantes elásticas da madeira.

Calegari (2006) cita que a propagação de ondas ultrassônicas se dá por meio de um circuito eletrônico que emite pulsos elétricos. Esses pulsos são conduzidos por cabos coaxiais e convertidos em ondas elásticas pelo cristal piezoelétrico, localizado nos transdutores. As vibrações mecânicas se deslocam pelo material, que atenua o sinal emitido pelo gerador. $\mathrm{O}$ sinal retardado é recuperado por outro cristal piezoelétrico, sendo então amplificados e transformados em pulsos elétricos novamente, permitindo a medição do tempo de propagação. A partir da distância e do tempo de percurso da onda sonora, calcula-se a sua velocidade.

Diversos fatores podem influenciar a propagação de ondas na madeira, tais como o teor de umidade, as propriedades anatômicas (espécie), físicas (massa específica) e morfológicas (tipos de lenhos e ângulo da grã), a presença de defeitos (nós e rachaduras), as condições do meio (temperatura e umidade relativa), as características dos transdutores e os tipos de ondas (KAWAMOTO; WILLIAMS, 2002; BUCUR; BÖNHKE, 1994; CARRASCO; AZEVEDO JÚNIOR, 2003; OLIVEIRA et al., 2005).

Segundo Calegari et al. (2008), os tipos de transdutores mais comuns são os de faces planas, que requerem o uso de acoplantes (óleo, gel, graxa ou vaselina), sendo aplicados entre a superfície da peça em ensaio e o transdutor, a fim de reduzir erros, uma vez que a ocorrência de ar entre esses meios implica maior tempo de propagação da onda (velocidade da onda ultrassônica é menor no ar), reduzindo sua velocidade.

Como desvantagens de uso dos transdutores de faces planas, Nesvijski (2003) descreveu que os acoplantes proporcionam instabilidade das repetições durante os testes, que não podem ser utilizados em superfícies ásperas ou curvas e que as alterações da distância entre os transdutores emissores-receptores aumentam as possibilidades de erro. Segundo o mesmo autor, esses problemas poderiam ser evitados pela estabilização do contato durante os testes, utilizando-se transdutores de pontos secos, o que pode ser possível pelo uso de extensores de ondas. Devido à rapidez, seu uso permite ainda aumentar a precisão dos dados pelo aumento do número de repetições (LORENZI, 2000).

Os principais tipos de ondas para a determinação das propriedades da madeira são as de volume e as de superfície (OLIVEIRA et al., 2005). Entre as ondas de volume, encontram-se ondas transversais, nas quais a vibração das partículas ocorre na direção perpendicular à de propagação, e ondas longitudinais, cujas partículas do meio em que se propagam vibram na mesma direção de propagação da onda nesse meio. Por sua vez, as ondas de superfície podem ser do tipo Rayleigh, Love e Lamb. A principal característica é de apresentarem movimento elíptico e ocorrerem, exclusivamente, na superfície dos sólidos.

Um dos principais fatores que determinam o tipo de onda são as características (modelo) dos transdutores. Nesse sentido, durante as análises não-destrutivas, é possível que se obtenham resultados diferenciados quanto à precisão e sensibilidade, devido ao tipo de onda gerada por diferentes transdutores do aparelho de ultrassom. Desse modo, o presente trabalho teve como objetivo avaliar os tipos de ondas 
geradas por dois modelos de transdutores empregados na determinação do módulo de elasticidade dinâmico por meio de ultrassom e correlacioná-los ao módulo de elasticidade estático obtido mediante ensaio destrutivo de compressão paralela às fibras.

\section{MATERIAL E MÉTODOS}

\section{Coleta e preparo de material}

Para realização dos ensaios, foram utilizadas madeiras de Eucalyptus grandis W. Hill ex Maiden, Patagonula americana L., Pinus elliottii Engelm. e Araucaria angustifolia (Bertol.) Kuntze, as quais possuíam aproximadamente $15,35,40$ e 50 anos de idade, respectivamente, obtidas de plantios homogêneos localizados nos municípios de Santa Maria, Passo Fundo e São Francisco de Paula, estado do Rio Grande do Sul.

De modo aleatório, foram selecionadas árvores adultas de bom fuste, com grã reta e diâmetro à altura do peito (DAP) de aproximadamente $40 \mathrm{~cm}$. De cada espécie foram coletadas três árvores, das quais foram retiradas as duas primeiras toras, $\mathrm{com} 200 \mathrm{~cm}$ de comprimento cada.

Logo após o corte das árvores, de cada tora retiraram-se duas pranchas de 5,2 $\mathrm{cm}$ de espessura, paralelas à medula, sendo uma localizada acima e a outra abaixo desta. De cada prancha, obtiveram-se quatro peças de $5,2 \mathrm{~cm}$ de largura, sendo duas para cada região, próxima à medula e próxima à casca (Figura 1).

As peças foram seccionadas, dando origem a amostras de dimensões nominais de $5,2 \mathrm{~cm}$ de espessura x 5,2 cm de largura x $20 \mathrm{~cm}$ de comprimento. As amostras que apresentavam defeitos, como grande inclinação da grã, presença de nós e medula, foram descartadas.

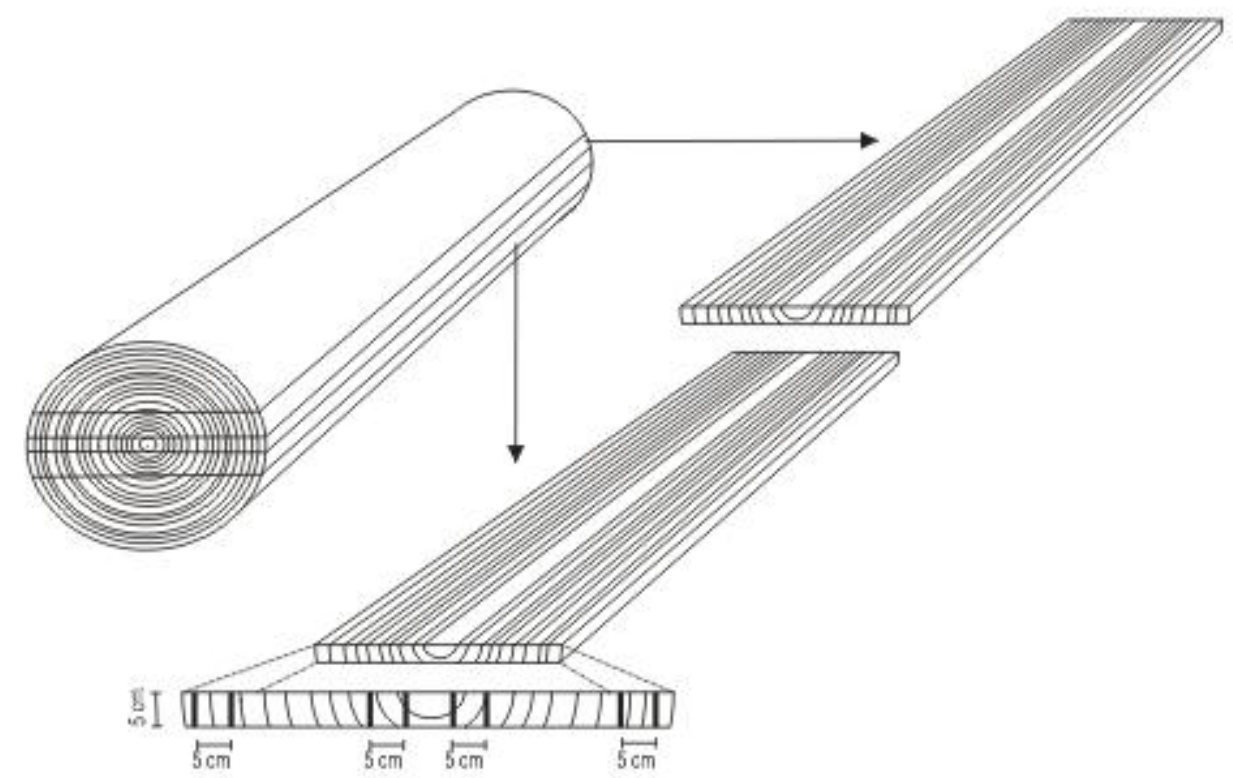

Figura 1. Esquema de corte das toras e retirada das tábuas.

Figure 1. Scheme of cut of logs and removed of the boards.

\section{Acondicionamento do material}

Antecedendo aos ensaios destrutivos e não-destrutivos, as amostras foram acondicionadas em uma câmara climatizada $\left(20 \pm 3{ }^{\circ} \mathrm{C}\right.$ de temperatura e $65 \pm 5 \%$ de umidade relativa) até atingirem teor de umidade de equilíbrio de 12\%, de modo a atender as normas da ASTM D143-94 (1995). Finalizado o processo, as amostras foram lixadas na seção transversal, obtendo-se dimensões nominais de $5 \times 5 \times 20$ (espessura, largura e comprimento). Posteriormente, foi determinada a massa específica aparente por meio da massa e das dimensões das amostras a $12 \%$ de umidade, com auxílio de balança eletrônica e paquímetro digital, precisões de $0,01 \mathrm{~g}$ e $0,01 \mathrm{~mm}$, respectivamente. 


\section{Delineamento experimental}

As amostras de madeira próxima à medula e à casca das quatro espécies florestais foram submetidas a três métodos de ensaios, de compressão paralela e de ultrassom (transdutores de faces planas e de pontos secos), para determinação do módulo de elasticidade. Desse modo, os tratamentos foram baseados na combinação das quatro espécies, duas regiões da madeira e três métodos de ensaios (Tabela 1), totalizando 24 tratamentos.

Tabela 1. Delineamento dos tratamentos do experimento.

Table 1. Design of experiment treatments.

\begin{tabular}{|c|c|c|c|c|}
\hline Tratamento & Espécie & Região & Métodos de avaliação & $\mathrm{N}^{0}$. de amostras \\
\hline 1 & Eucalyptus grandis & Próxima à medula & Destrutivo & 26 \\
\hline 2 & Eucalyptus grandis & Próxima à medula & Não-destrutivo - Face plana & 26 \\
\hline 3 & Eucalyptus grandis & Próxima à medula & Não-destrutivo - Ponto seco & 26 \\
\hline 4 & Eucalyptus grandis & Próxima à casca & Destrutivo & 30 \\
\hline 5 & Eucalyptus grandis & Próxima à casca & Não-destrutivo - Face plana & 30 \\
\hline 6 & Eucalyptus grandis & Próxima à casca & Não-destrutivo - Ponto seco & 30 \\
\hline 7 & Patagonula americana & Próxima à medula & Destrutivo & 30 \\
\hline 8 & Patagonula americana & Próxima à medula & Não-destrutivo - Face plana & 30 \\
\hline 9 & Patagonula americana & Próxima à medula & Não-destrutivo - Ponto seco & 30 \\
\hline 10 & Patagonula americana & Próxima à casca & Destrutivo & 30 \\
\hline 11 & Patagonula americana & Próxima à casca & Não-destrutivo - Face plana & 30 \\
\hline 12 & Patagonula americana & Próxima à casca & Não-destrutivo - Ponto seco & 30 \\
\hline 13 & Araucaria angustifolia & Próxima à medula & Destrutivo & 26 \\
\hline 14 & Araucaria angustifolia & Próxima à medula & Não-destrutivo - Face plana & 26 \\
\hline 15 & Araucaria angustifolia & Próxima à medula & Não-destrutivo - Ponto seco & 26 \\
\hline 16 & Araucaria angustifolia & Próxima à casca & Destrutivo & 30 \\
\hline 17 & Araucaria angustifolia & Próxima à casca & Não-destrutivo - Face plana & 30 \\
\hline 18 & Araucaria angustifolia & Próxima à casca & Não-destrutivo - Ponto seco & 30 \\
\hline 19 & Pinus elliottii & Próxima à medula & Destrutivo & 30 \\
\hline 20 & Pinus elliottii & Próxima à medula & Não-destrutivo - Face plana & 30 \\
\hline 21 & Pinus elliottii & Próxima à medula & Não-destrutivo - Ponto seco & 30 \\
\hline 22 & Pinus elliottii & Próxima à casca & Destrutivo & 30 \\
\hline 23 & Pinus elliottii & Próxima à casca & Não-destrutivo - Face plana & 30 \\
\hline 24 & Pinus elliottii & Próxima à casca & Não-destrutivo - Ponto seco & 30 \\
\hline
\end{tabular}

\section{Ensaios não-destrutivos}

Para a realização dos ensaios não-destrutivos, foi utilizado o equipamento de ultrassom PUNDIT (Portable Ultrasonic Non-destructive Testing), fabricado pela CNC Electronic, Inglaterra. Esse aparelho é dotado de dois modelos de transdutores, de faces planas e de pontos secos, com frequência de $50 \mathrm{kHz}$, que medem diretamente o tempo de propagação da onda, em microssegundos ( $\mu \mathrm{s})$.

A determinação do tempo de propagação das ondas ultrassônicas obtidas por meio de transdutores de faces planas foi realizada no centro das amostras, considerando a direção longitudinal da madeira. Os transdutores utilizados nos ensaios apresentavam diâmetro útil de $2,5 \mathrm{~cm}$, sendo acoplados nos topos das peças ensaiadas (Figura 2 A). Para garantir o melhor contato entre a madeira e os transdutores, aplicou-se vaselina em pasta nos topos das peças ensaiadas.

Por sua vez, os transdutores de pontos secos eram constituídos por extensores de ondas cônicos e acoplados em suporte, que também era utilizado para apoio das mãos. Esse conjunto (suporte e transdutores com extensores de ondas) é um dispositivo patenteado, sendo que o ângulo dos transdutores com relação à vertical apresenta valor fixo de $30^{\circ}$. De modo semelhante ao uso dos transdutores de faces planas, o tempo de propagação das ondas ultrassônicas foi obtido no centro das amostras, considerando a direção longitudinal da madeira. Uma reta foi demarcada para que ambos os pontos secos ficassem perfeitamente alinhados, sendo então pressionados sobre a reta, apenas em contato superficial (Figura 2 B).

Para cada modelo de transdutor, foram realizadas duas leituras do tempo de propagação das ondas. A partir da razão entre a média das leituras e o comprimento do trecho percorrido pelas ondas, 
efetuou-se o cálculo da velocidade de propagação. Para os transdutores de faces planas, o comprimento do trecho percorrido pelas ondas foi igual ao comprimento real de cada amostra ensaiada. Por sua vez, para os transdutores de pontos secos, foi igual à distância entre os pontos de contato $(12 \mathrm{~cm})$. A constante elástica dinâmica foi determinada por meio do produto entre a velocidade de propagação das ondas e a massa específica (Equação 1).
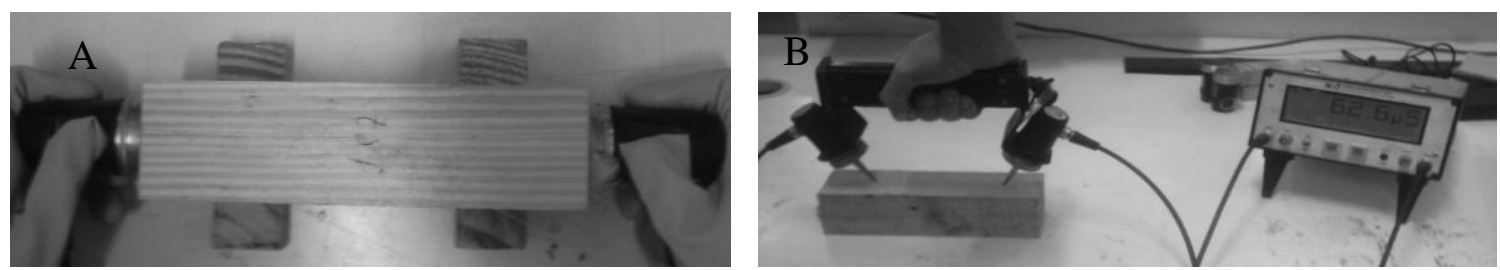

Figura 2. Determinação do tempo de propagação das ondas ultrassônicas na madeira utilizando transdutores de faces planas (A) e de pontos secos (B).

Figure 2. Determination of time of propagation of the ultrasonic waves in the wood using planes faces (A) and point-contact transducers (B).

$$
C_{L L}=M E * V^{2}
$$

Em que: $\mathrm{C}_{\mathrm{LL}}=$ constante elástica dinâmica $(\mathrm{MPa})$;

$\mathrm{V}=$ velocidade de propagação da onda $\left(\mathrm{m} \cdot \mathrm{s}^{-1}\right)$;

$\mathrm{ME}=$ massa específica aparente a $12 \%$ de umidade $\left(\mathrm{kg} \cdot \mathrm{m}^{-3}\right)$.

\section{Ensaios destrutivos}

Para avaliar o comportamento dos tipos de ondas gerados pelos transdutores utilizados, as mesmas amostras foram submetidas a ensaios destrutivos de compressão paralela às fibras, em máquina universal de ensaios da marca Wolpert-Amsler, atendendo, no geral, às prescrições da American Society for Testing and Materials - ASTM D143-94 (1995).

\section{Análise estatística}

Os resultados foram interpretados utilizando-se pacote estatístico Statgraphics Plus ${ }^{\circledR}$, com auxílio de testes de comparação de médias (LSD Fisher, $p>0,05$ ) e análises de regressões lineares, em que a variável independente foi a constante elástica dinâmica obtida em ensaio de ultrassom com os dois modelos de transdutores, e a variável dependente foi o módulo de elasticidade estático obtido em ensaio convencional de compressão paralela às fibras.

\section{RESULTADOS E DISCUSSÃO}

Os valores médios da velocidade de propagação das ondas ultrassônicas obtidas com o uso dos transdutores de faces planas foram bastante próximos aos relatados por outros pesquisadores (BALLARIN; NOGUEIRA, 2005; CARRASCO; AZEVEDO JÚNIOR, 2003; PUCCINI, 2002; SIMPSON, 1998; GONÇALEZ et al., 2001), situando-se entre 4.000 e $6.000 \mathrm{~m} . \mathrm{s}^{-1}$ (Tabela 2). Tanto neste estudo como nos demais citados, as ondas geradas pelo aparelho de ultrassom foram longitudinais, por isso a semelhança entre os valores.

Por sua vez, as ondas geradas pelos transdutores de pontos secos foram do tipo superficial, resultando em valores médios da velocidade de aproximadamente metade do observado com os transdutores de faces planas, que geraram ondas longitudinais. Calegari (2006), realizando o monitoramento da redução do conteúdo de umidade de madeiras, com auxílio de transdutores de pontos secos e de faces planas, expostas a secagem, e Lorenzi (2000), avaliando estruturas de concreto por meio de testes ultrassônicos, verificaram comportamento semelhante ao registrado neste estudo.

De acordo com Nesvijski (2003), transdutores de pontos secos não geram ondas direcionadas, proporcionando maior tempo de propagação quando comparadas às ondas geradas por meio de transdutores de faces planas. Além desse fator, a forma, o material de fabricação e/ou o ângulo de inclinação do extensor de ondas também contribuem para a alteração do tipo de onda gerada pelo transdutor, alterando também sua velocidade de propagação. 
Tabela 2. Velocidade de propagação das ondas ultrassônicas e constante elástica dinâmica obtidas com transdutores de faces planas e de pontos secos, e massa específica para as amostras de madeira próxima à medula e à casca das quatro espécies.

Table 2. Speed of propagation of the ultrasonic waves and dynamic elastic constant obtained with planes faces and point-contact transducers, and density for the samples of wood near the pith and the bark of four species.

\begin{tabular}{|c|c|c|c|c|c|c|c|c|c|c|}
\hline & \multicolumn{5}{|c|}{ Próxima à medula } & \multicolumn{5}{|c|}{ Próxima à casca } \\
\hline & \multirow[b]{2}{*}{ ME } & \multicolumn{2}{|c|}{ TFP } & \multicolumn{2}{|c|}{ TPS } & \multirow[b]{2}{*}{ ME } & \multicolumn{2}{|c|}{ TFP } & \multicolumn{2}{|c|}{ TPS } \\
\hline & & $\mathbf{V}_{\text {SOM }}$ & $\mathrm{C}_{\mathrm{LL}}$ & $\mathbf{V}_{\text {SOM }}$ & $\mathrm{C}_{\mathrm{LL}}$ & & $\mathbf{V}_{\text {SOM }}$ & $\mathrm{C}_{\mathrm{LL}}$ & $\mathbf{V}_{\text {SOM }}$ & $\mathrm{C}_{\mathrm{LL}}$ \\
\hline \multicolumn{11}{|c|}{ Eucalyptus grandis } \\
\hline Mínimo & 0,499 & 4.890 & 13.255 & 1.905 & 2.259 & 0,594 & 4.762 & 16.899 & 1.863 & 2.779 \\
\hline Máximo & 0,654 & 5.464 & 18.404 & 2.553 & 3.863 & 0,835 & 5.797 & 22.837 & 2.597 & 4.477 \\
\hline Média & 0,586 & 5.180 & 15.698 & 2.352 & 3.238 & 0,721 & 5.269 & 19.884 & 2.315 & 3.843 \\
\hline $\mathrm{CV}(\%)$ & 6,76 & 2,79 & 6,44 & 5,26 & 9,65 & 10,74 & 5,25 & 6,84 & 5,78 & 9,05 \\
\hline \multicolumn{11}{|c|}{ Patagonula americana } \\
\hline Mínimo & 0,698 & 4.323 & 14.259 & 1.767 & 2.397 & 0,675 & 4.418 & 14.259 & 2.174 & 3.322 \\
\hline Máximo & 0,815 & 5.074 & 19.651 & 2.317 & 4.177 & 0,764 & 5.000 & 19.103 & 2.339 & 4.038 \\
\hline Média & 0,743 & 4.671 & 16.245 & 2.209 & 3.635 & 0,721 & 4.868 & 17.101 & 2.272 & 3.722 \\
\hline CV $(\%)$ & 4,08 & 4,81 & 10,53 & 5,24 & 10,47 & 2,77 & 2,98 & 6,56 & 1,89 & 4,51 \\
\hline \multicolumn{11}{|c|}{ Araucaria angustifolia } \\
\hline Mínimo & 0,441 & 5.057 & 11.265 & 2.308 & 2.646 & 0,447 & 5.132 & 12.916 & 2.308 & 2.783 \\
\hline Máximo & 0,579 & 5.556 & 16.907 & 2.545 & 3.483 & 0,591 & 6.334 & 22.525 & 2.721 & 4.219 \\
\hline Média & 0,519 & 5.367 & 14.976 & 2.455 & 3.129 & 0,524 & 6.062 & 19.379 & 2.576 & 3.490 \\
\hline CV (\%) & 6,61 & 2,08 & 8,69 & 2,38 & 7,06 & 7,14 & 5,33 & 14,40 & 5,43 & 13,11 \\
\hline \multicolumn{11}{|c|}{ Pinus elliottii } \\
\hline Mínimo & 0,405 & 3.534 & 5.901 & 1.843 & 1.578 & 0,610 & 5.063 & 16.206 & 2.044 & 2.949 \\
\hline Máximo & 0,596 & 5.571 & 17.652 & 2.985 & 4.781 & 0,728 & 6.024 & 24.470 & 2.564 & 4.787 \\
\hline Média & 0,489 & 4.600 & 10.501 & 2.226 & 2.459 & 0,671 & 5.613 & 21.204 & 2.359 & 3.744 \\
\hline CV (\%) & 9,41 & 12,80 & 27,12 & 11,48 & 27,55 & 4,89 & 5,21 & 11,27 & 4,97 & 10,91 \\
\hline
\end{tabular}

Apesar de este trabalho ter apresentado baixa velocidade de propagação das ondas ultrassônicas pelo emprego de transdutores de pontos secos, resultados de outras pesquisas (PUCCINI, 2002; OLIVEIRA et al., 2002; COSTA, 2005) descrevem altos valores de velocidade obtidos por transdutores de pontos secos, próximos aos observados por transdutores de faces planas. Nesses estudos, os autores obtiveram ondas longitudinais, por isso a semelhança. Os baixos resultados obtidos neste trabalho podem sugerir que as ondas não eram efetivamente longitudinais, o que foi ocasionado pelo ângulo formado entre os transdutores e a superfície da madeira. Uma onda, ao incidir em ângulo com as fibras, não gera onda longitudinal pura, ou seja, há componentes longitudinais e transversais, a não ser que o ângulo seja calculado para gerar uma onda puramente longitudinal. No presente estudo, o ângulo pode ter proporcionado a formação de uma onda de superfície, que apresenta baixa velocidade de propagação, principalmente considerando a pequena distância entre os transdutores, conforme sugerido por Calegari et al. (2008).

Mediante testes de médias, verifica-se que os valores, tanto de velocidade de propagação das ondas quanto de constante elástica dinâmica, obtidos pelos dois modelos de transdutores apresentaram diferença significativa a 95\% de probabilidade (Tabela 3). Além disso, observa-se que o uso dos transdutores de faces planas foi mais sensível, em comparação ao uso dos transdutores de pontos secos, à diferenciação entre as duas regiões das madeiras ensaiadas, exceto para a madeira de Eucalyptus grandis quando considerada a velocidade de propagação das ondas.

A madeira adulta, próxima à casca, apresenta maior percentual de lenho tardio, fibras mais espessas, menor diâmetro de lume e é mais densa, proporcionando maior velocidade das ondas ultrassonoras. O contrário ocorre com a madeira juvenil, próxima à medula (SHIMOYAMA, 2005). 
Bucur (1995) salientou que a velocidade das ondas é maior na madeira próxima à casca, porém a maior ascensão é verificada na madeira próxima à medula, aumentando significativamente nos primeiros anos de crescimento da árvore e, posteriormente, se estabilizando durante a formação da madeira adulta.

Tabela 3. Valores de velocidade de propagação das ondas ultrassônicas e constante elástica dinâmica submetidos aos testes de médias.

Table 3. Values of speed of propagation of the ultrasonic waves and dynamic elastic constant submitted tests of means.

\begin{tabular}{lcc}
\hline Tipo de madeira e tipo de transdutor & $\mathbf{V}_{\text {SOM }}\left(\mathbf{m . s}^{-\mathbf{1}}\right)$ & $\mathbf{C}_{\mathbf{L L}}(\mathbf{M P a})$ \\
\hline Próximo à medula - TFP & Eucalyptus & grandis \\
Próximo à casca - TFP & $5.180 \mathrm{a}$ & $15.698 \mathrm{~b}$ \\
Próximo à medula - TPS & $5.269 \mathrm{a}$ & $19.884 \mathrm{a}$ \\
Próximo à casca - TPS & $2.352 \mathrm{~b}$ & $3.238 \mathrm{~d}$ \\
\hline & $2.315 \mathrm{~b}$ & $3.843 \mathrm{c}$ \\
\hline Próximo à medula - TFP & Patagonula americana & $16.245 \mathrm{~b}$ \\
Próximo à casca - TFP & $4.671 \mathrm{~b}$ & $17.101 \mathrm{a}$ \\
Próximo à medula - TPS & $4.868 \mathrm{a}$ & $3.635 \mathrm{c}$ \\
Próximo à casca - TPS & $2.209 \mathrm{c}$ & $3.722 \mathrm{c}$ \\
\hline & $2.272 \mathrm{c}$ & \\
\hline & Araucaria angustifolia & $14.976 \mathrm{~b}$ \\
Próximo à medula - TFP & $5.367 \mathrm{~b}$ & $19.379 \mathrm{a}$ \\
Próximo à casca - TFP & $6.062 \mathrm{a}$ & $3.129 \mathrm{c}$ \\
Próximo à medula - TPS & $2.455 \mathrm{~d}$ & $3.490 \mathrm{c}$ \\
Próximo à casca - TPS & $2.576 \mathrm{c}$ & $10.501 \mathrm{~b}$ \\
\hline & Pinus elliottii \\
\hline Próximo à medula - TFP & $4.600 \mathrm{~b}$ & $21.204 \mathrm{a}$ \\
Próximo à casca - TFP & $5.613 \mathrm{a}$ & $2.459 \mathrm{~d}$ \\
Próximo à medula - TPS & $2.226 \mathrm{c}$ & $3.744 \mathrm{c}$ \\
Próximo à casca - TPS & $2.359 \mathrm{c}$ &
\end{tabular}

Médias seguidas por letras iguais não diferem significativamente entre si (LSD Fisher, $p>0,05$ ).

Ao analisar a Equação 1, a velocidade de propagação das ondas é inversamente proporcional à raiz quadrada da massa específica da madeira, ou seja, pela teoria, as coníferas tenderiam a apresentar maiores velocidades de propagação em comparação às folhosas, conforme verificado neste estudo. Para Carrasco; Azevedo Júnior (2003), não é propriamente o aumento da massa específica que acarreta o aumento na velocidade de propagação das ondas em madeiras. Segundo os autores, o aumento da massa específica pode decorrer da maior deposição de celulose na face interna da parede celular. Essa deposição acarreta aumento mais significativo nos valores de rigidez do que nos valores de massa específica da madeira. Dessa forma, mesmo que haja aumento da massa específica, a velocidade não diminui, pois é compensada pelo apreciável aumento da rigidez.

A explicação para as maiores velocidades de propagação das ondas em coníferas, tanto com o uso de transdutores de faces planas quanto de pontos secos, está relacionada com as propriedades anatômicas da madeira. Bucur (1988) cita que, considerando o sentido longitudinal da madeira, o aumento do comprimento das fibras favorece a propagação, devido ao maior caminho contínuo da parede a ser percorrido pelas ondas. Assim, a contínua e uniforme estrutura das coníferas favorece o aumento da velocidade das ondas quando comparada às folhosas.

Os valores das constantes elásticas dinâmicas obtidas por meio de transdutores de faces planas (ondas longitudinais) para as madeiras próximas à medula e à casca foram maiores que os valores dos módulos de elasticidade à compressão paralela (Tabelas 2 e 4), confirmando os resultados apresentados por Bartholomeu (2001), Nogueira; Ballarin (2002) e Puccini (2002). De acordo com Ouis (2002), e considerando a natureza viscoelástica da madeira, quanto maior a frequência de excitação da fonte, maior é o valor inferido para o módulo de elasticidade dinâmico. No entanto, as constantes elásticas dinâmicas obtidas pelos ensaios com transdutores de pontos secos apresentaram valores bastante inferiores aos verificados nos ensaios de compressão paralela. $\mathrm{O}$ ângulo formado entre os transdutores e a superfície da madeira gerou ondas de superfície com baixa velocidade de propagação, prejudicando a obtenção de resultados mais próximos ao registrado destrutivamente. 
Tabela 4. Módulo de elasticidade estático obtido por meio de ensaio de compressão paralela às fibras.

Table 4. Obtained static modulus of elasticity by means of test of parallel compression.

\begin{tabular}{|c|c|c|c|c|}
\hline & Próxima à medula & Próxima à casca & Próxima à medula & Próxima à casca \\
\hline & $\mathrm{E}_{\mathrm{C} 0}(\mathrm{MPa})$ & $\mathrm{E}_{\mathrm{C} 0}(\mathrm{MPa})$ & $\mathrm{E}_{\mathrm{C} 0}(\mathrm{MPa})$ & $\mathrm{E}_{\mathrm{C} 0}(\mathrm{MPa})$ \\
\hline & \multicolumn{2}{|c|}{ Eucalyptus grandis } & \multicolumn{2}{|c|}{ Patagonula americana } \\
\hline Mínimo & 10.965 & 14.557 & 10.560 & 12.000 \\
\hline Máximo & 14.880 & 19.859 & 16.400 & 16.188 \\
\hline Médio & 13.119 & 16.944 & 13.570 & 14.616 \\
\hline \multirow[t]{2}{*}{$\mathrm{CV}(\%)$} & 7,90 & 8,13 & 13,21 & 7,13 \\
\hline & \multicolumn{2}{|c|}{ Araucaria angustifolia } & \multicolumn{2}{|c|}{ Pinus elliottii } \\
\hline Mínimo & 8.344 & 9.631 & 3.803 & 12.617 \\
\hline Máximo & 12.360 & 16.712 & 16.840 & 23.712 \\
\hline Médio & 10.897 & 14.367 & 8.739 & 17.866 \\
\hline $\mathrm{CV}(\%)$ & 8,89 & 14,02 & 32,36 & 14,92 \\
\hline
\end{tabular}

$\mathrm{E}_{\mathrm{C} 0}=$ Módulo de elasticidade estático.

Na tabela 5 são apresentadas as equações ajustadas para a estimativa do módulo de elasticidade estático à compressão paralela em função da constante elástica dinâmica obtida pelos transdutores de faces planas e de pontos secos. Verifica-se que os modelos estatísticos apresentaram valores significativos a 1\%, em termos dos parâmetros da regressão, exceto o modelo ajustado em função da constante elástica dinâmica obtida por transdutores de pontos secos para madeira próxima à medula de Eucalyptus grandis. Além disso, os melhores modelos (maior $\mathrm{R}_{\mathrm{aj}}{ }^{2}$, menor $\mathrm{S}_{\mathrm{yx}}$ e maior $\mathrm{F}_{\text {calc. }}$.) foram observados para os dados ajustados com base na constante elástica dinâmica obtida pelos transdutores de faces planas.

Tabela 5. Equações ajustadas para a estimativa do módulo de elasticidade estático à compressão paralela em razão da constante elástica dinâmica obtida pelos dois modelos de transdutores.

Table 5. Equations adjusted to estimate of the static modulus of elasticity (test of parallel compression) in reason of the elastic constant dynamics obtained for the different transducers.

\begin{tabular}{|c|c|c|c|c|c|}
\hline $\begin{array}{l}\text { Espécie - Lenho - } \\
\text { Transdutor }\end{array}$ & Função & Equação de regressão & $\mathbf{R}_{\mathrm{aj}}^{2}(\%)$ & $\mathbf{S}_{\mathrm{yx}}$ & $\mathbf{F}_{\text {calc. }}$ \\
\hline EG - PM - TFP & $\mathrm{E}_{\mathrm{C} 0}=\mathrm{f}\left(\mathrm{C}_{\mathrm{LL}}\right)$ & $\mathrm{E}_{\mathrm{c} 0}=-258,477+0,852187 * \mathrm{C}_{\mathrm{LL}}$ & 67,65 & 589,81 & $53,28 * *$ \\
\hline EG - PC - TFP & $\mathrm{E}_{\mathrm{C} 0}=\mathrm{f}\left(\mathrm{C}_{\mathrm{LL}}\right)$ & $\mathrm{E}_{\mathrm{c} 0}=-1815,77+0,943475 * \mathrm{C}_{\mathrm{LL}}$ & 86,19 & 511,96 & $182,01 * *$ \\
\hline EG - PM - TPS & $\mathrm{E}_{\mathrm{C} 0}=\mathrm{f}\left(\mathrm{C}_{\mathrm{LL}}\right)$ & $\mathrm{E}_{\mathrm{c} 0}=12840,1+0,0860453 * \mathrm{C}_{\mathrm{LL}}$ & 0,07 & 1058,04 & $0,02^{\mathrm{NS}}$ \\
\hline EG - PC - TPS & $\mathrm{E}_{\mathrm{C} 0}=\mathrm{f}\left(\mathrm{C}_{\mathrm{LL}}\right)$ & $\mathrm{E}_{\mathrm{c} 0}=7093,81+2,56324 * \mathrm{C}_{\mathrm{LL}}$ & 39,83 & 1068,67 & $20,20 * *$ \\
\hline PA - PM - TFP & $\mathrm{E}_{\mathrm{C} 0}=\mathrm{f}\left(\mathrm{C}_{\mathrm{LL}}\right)$ & $\mathrm{E}_{\mathrm{c} 0}=-2154,89+0,96802 * \mathrm{C}_{\mathrm{LL}}$ & 84,45 & 707,14 & $104,16^{* *}$ \\
\hline PA - PC - TFP & $\mathrm{E}_{\mathrm{C} 0}=\mathrm{f}\left(\mathrm{C}_{\mathrm{LL}}\right)$ & $\mathrm{E}_{\mathrm{c} 0}=-348,77+0,87507 * \mathrm{C}_{\mathrm{LL}}$ & 87,98 & 361,61 & $140^{* *}$ \\
\hline PA - PM - TPS & $\mathrm{E}_{\mathrm{C} 0}=\mathrm{f}\left(\mathrm{C}_{\mathrm{LL}}\right)$ & $\mathrm{E}_{\mathrm{c} 0}=2755,46+2,97558 * \mathrm{C}_{\mathrm{LL}}$ & 36,56 & 1428,13 & $11,95^{* *}$ \\
\hline PA - PC - TPS & $\mathrm{E}_{\mathrm{C} 0}=\mathrm{f}\left(\mathrm{C}_{\mathrm{LL}}\right)$ & $\mathrm{E}_{\mathrm{c} 0}=-667,614+4,1062 * \mathrm{C}_{\mathrm{LL}}$ & 40,61 & 803,64 & $13,99^{* *}$ \\
\hline AA - PM - TFP & $\mathrm{E}_{\mathrm{C} 0}=\mathrm{f}\left(\mathrm{C}_{\mathrm{LL}}\right)$ & $\mathrm{E}_{\mathrm{c} 0}=240,685+0,71155^{*} \mathrm{C}_{\mathrm{LL}}$ & 91,08 & 289,36 & $327,58^{* *}$ \\
\hline AA - PC - TFP & $\mathrm{E}_{\mathrm{C} 0}=\mathrm{f}\left(\mathrm{C}_{\mathrm{LL}}\right)$ & $\mathrm{E}_{\mathrm{c} 0}=716,634+0,70435^{*} \mathrm{C}_{\mathrm{LL}}$ & 94,99 & 450,82 & $551,16^{* *}$ \\
\hline AA - PM - TPS & $\mathrm{E}_{\mathrm{C} 0}=\mathrm{f}\left(\mathrm{C}_{\mathrm{LL}}\right)$ & $\mathrm{E}_{\mathrm{c} 0}=-212,306+3,5503 * \mathrm{C}_{\mathrm{LL}}$ & 64,42 & 577,811 & $58,93^{* *}$ \\
\hline AA - PC - TPS & $\mathrm{E}_{\mathrm{C} 0}=\mathrm{f}\left(\mathrm{C}_{\mathrm{LL}}\right)$ & $\mathrm{E}_{\mathrm{C} 0}=2952,89+3,27041 * \mathrm{C}_{\mathrm{LL}}$ & 53,56 & 1372,9 & $34,45^{* *}$ \\
\hline PE - PM - TFP & $\mathrm{E}_{\mathrm{C} 0}=\mathrm{f}\left(\mathrm{C}_{\mathrm{LL}}\right)$ & $\mathrm{E}_{\mathrm{c} 0}=-1321,31+0,957967 * \mathrm{C}_{\mathrm{LL}}$ & 92,82 & 758,01 & $375,69 * *$ \\
\hline PE - PC - TFP & $\mathrm{E}_{\mathrm{C} 0}=\mathrm{f}\left(\mathrm{C}_{\mathrm{LL}}\right)$ & $\mathrm{E}_{\mathrm{c} 0}=-2676,24+0,968767 * \mathrm{C}_{\mathrm{LL}}$ & 74,50 & 1346,27 & $85,72^{* *}$ \\
\hline PE - PM - TPS & $\mathrm{E}_{\mathrm{C} 0}=\mathrm{f}\left(\mathrm{C}_{\mathrm{LL}}\right)$ & $\mathrm{E}_{\mathrm{c} 0}=1441,05+2,96737 * \mathrm{C}_{\mathrm{LL}}$ & 48,76 & 2024,4 & $28,60 * *$ \\
\hline PE - PC - TPS & $\mathrm{E}_{\mathrm{C} 0}=\mathrm{f}\left(\mathrm{C}_{\mathrm{LL}}\right)$ & $\mathrm{E}_{\mathrm{C} 0}=3732,04+3,77528 * \mathrm{C}_{\mathrm{LL}}$ & 31,05 & 2213,7 & $14,06^{* *}$ \\
\hline
\end{tabular}

EG: Eucalyptus grandis; PA: Patagonula americana; AA: Araucaria angustifolia; PE: Pinus elliottii; PM: próxima à medula; PC: próxima à casca; $\mathrm{R}_{\mathrm{aj}}{ }^{2}$ : coeficiente de determinação ajustado; $\mathrm{S}_{\mathrm{yx}}$ : erro padrão da estimativa; $\mathrm{F}$ : valor de $\mathrm{F}$ calculado; f: função; **: significativo a $1 \%$ de probabilidade; ${ }^{\text {NS }}$ : não significativo. 
A reduzida área superficial de contato dos transdutores de pontos secos com a madeira pode ter contribuído para as menores correlações da constante elástica dinâmica e o módulo de elasticidade à compressão paralela, visto que a velocidade de propagação das ondas ultrassônicas pode ser influenciada pela região, por exemplo, lenho inicial ou tardio (FEENEY et al., 1998), em que os transdutores de pontos secos são posicionados, além do fato de as ondas geradas serem superficiais.

\section{CONCLUSÕES}

Com base nos resultados obtidos, pode-se concluir que:

- As ondas longitudinais geradas pelos transdutores de faces planas são mais sensíveis às diferenças de região da madeira (próxima à casca e próxima à medula), tornando-as ideais para ensaios de caracterização da madeira no que diz respeito à qualidade.

- A constante elástica dinâmica obtida pelo uso da onda longitudinal apresenta boa correlação com o módulo de elasticidade à compressão paralela, o que comprova a viabilidade da técnica, podendo esta ser expandida para avaliação de peças de maiores dimensões.

- Apesar dos transdutores de pontos secos serem mais práticos, não necessitando uso de acoplantes, as ondas geradas por esses instrumentos não são perfeitamente longitudinais, gerando ondas de superfície que apresentam baixa velocidade de propagação e consequentemente baixa constante elástica dinâmica.

\section{REFERÊNCIAS}

AMERICAN SOCIETY FOR TESTING AND MATERIALS - ASTM D 143. Standard methods of testing small clear specimens of timber. Annual Book of ASTM Standards, v. 04.10, p. 24-65, 1995.

BALLARIN, A. W.; NOGUEIRA, M. Determinação do módulo de elasticidade da madeira juvenil e adulta de Pinus taeda por ultrassom. Engenharia Agrícola, Jaboticabal, v. 25, n. 1, p. 19-28, 2005.

BARTHOLOMEU, A. Classificação de peças estruturais de madeira através do ultrassom. UNICAMP, 2001. 105 p. Tese (Doutorado em Engenharia Agrícola) - Universidade de Campinas, 2001.

BUCUR, V. Wood structure anisotropy estimated by acoustic invariants. IAWA Bulletin, Leiden, v. 9, n. 1, p. 67-74, 1988.

BUCUR, V. Acoustics of wood. New York: CRC Press Inc., 1995.

BUCUR, V.; BÖHNKE, I. Factors affecting ultrasonic measurements in solid wood. Ultrasonics. v. 32, n 5, p. 385-390, 1994.

CALEGARI, L. Uso da onda ultrassônica como meio de controle do processo de secagem da madeira. Santa Maria: UFSM, 2006. 91 p. Dissertação (Mestrado em Engenharia Florestal) Universidade Federal de Santa Maria, 2006.

CALEGARI, L.; STANGERLIN, D. M.; SANTINI, E. J.; HASELEIN, C. R.; GATTO, D. A.; CARMO, P. I. O.; SILVA FILHO, L. C. P. Avaliação de alguns fatores influentes na velocidade ultrassônica na madeira. Floresta, Curitiba, v. 38, n. 4, p. 607-615, 2008.

CARRASCO, E. V. M.; AZEVEDO JÚNIOR, A. P. Avaliação não-destrutiva de propriedades mecânicas de madeiras através de ultrassom - fundamentos físicos e resultados experimentais. Cerne, Lavras, v. 9, n. 2, p. 178-191, 2003.

COSTA, O. A. L. Velocidade de propagação de ondas de ultrassom na madeira para diferentes condições de umidade. 92 f. Tese (Doutorado em Engenharia Agrícola) - Universidade Estadual de Campinas, Campinas, SP, 2005.

FEENEY, F. E.; CHIVERS, R. C.; EVERTSEN, J. A.; KEATING, J. The influence of inhomogeneity on the propagation of ultrasound in wood. Ultrasonics, v. 36, n. 1, p. 449-453, 1998. 
GONÇALEZ, J. C.; VALLE, A. T.; COSTA, A. F. Estimativas das constantes elásticas da madeira por meio de ondas ultrassonoras (ultrassom). Cerne, Lavras, v. 7, n. 2, p. 81-92, 2001.

JANG, S. S. Evaluation of lumber properties by applying stress waves to larch logs grown in Korea. Forest Products Journal, Madison, v. 50, n. 3, p. 44-48, 2000.

JAYNE, B. A. Vibrational properties of wood. As indices of quality. Forest Products Journal, Madison, v. 9, n. 11, p. 413-416, 1959.

KABIR, M. F. Prediction of ultrasonic properties from grain angle. Journal of the Institute of Wood Science, v. 15, n. 5, p. 235-246, 2001.

KAWAMOTO, S.; WILLIANS, R. S. Acoustic emission and acoustic-ultrasonic techniques for wood and wood-based composites - A review. General Tecnical Report FPL-GTR-134. Madison: U.S. Department of Agriculture, Forest Service, Forest Products Laboratory, 2002. 16 p.

LORENZI, A. Aplicação de testes ultrassônicos para avaliação de estruturas de concreto utilizando elementos de inteligência artificial. 152 f. Dissertação (Mestrado em Engenharia Civil) - Universidade Federal de Santa Maria, Santa Maria, RS, 2000.

NESVIJSKI, E. G. Dry point contact transducers: design for new applications. In: The e-Journal of Nondestructive Testing, v. 9, n. 9, Berlin: NDT.net, 2003. Disponível em: <http://www.ndt.net/article/ v08n09/nesvi/nevsi.htm>. Acesso em 11/04/2009.

NOGUEIRA, M.; BALLARIN, A. W. Correlação entre módulos de elasticidade estático (flexão) e dinâmico (ultrassom) para algumas espécies de eucalipto. In: CONGRESSO IBERO-AMERICANO DE PESQUISA E DESENVOLVIMENTO DE PRODUTOS FLORESTAIS, 2., 2002, Curitiba. Anais... Curitiba: UFPR/FUPEF, 2002. 1 CD ROM.

OLIVEIRA, F. G. R.; CAMPOS, J. A. O.; PLETZ, E.; SALES, A. Nondestructive evaluation of wood using ultrasonic technique. Maderas. Ciencia y Tecnología, Concepción, v. 4, n. 2, p. 133-139, 2002.

OLIVEIRA, F. G. R.; CANDIAN, M.; LUCCHETTE, F. F.; SALGON, J. L.; SALES, A. Moisture content effect on ultrasonic velocity in Goupia glabra. Materials Research, São Carlos, v. 8, n. 1, p. 1114, 2005.

OUIS, D. Dispersion of wood as a consequence of its viscoelasticity. In: INTERNATIONAL SYMPOSIUM ON NONDESTRUCTIVE TESTING OF WOOD, 13., 2002, Berkeley. Proceedings... Berkeley: University of California, 2002. 8 p.

PUCCINI, C. T. Avaliação de aspectos de qualidade da madeira utilizando o ultrassom. Campinas: UNICAMP, 2002. 139 p. Tese (Doutorado em Engenharia Agrícola). Universidade Estadual de Campinas, 2002.

SHIMOYAMA, V. R. de S. Estimativas de propriedades da madeira de Pinus taeda através do método não-destrutivo emissão de ondas de tensão, visando à geração de produtos de alto valor agregado. Curitiba: UFPR, 2005. 151 p. Tese (Doutorado em Ciências Florestais) - Universidade Federal do Paraná, 2005.

SIMPSON, W. T. Relationship between speed of sound and moisture content of red oak and hard maple during drying. Wood and Fiber Science, Madison, v. 30, n. 4, p. 405-413, 1998.

WANG, X.; CARTER, P.; ROSS, R. J.; BRASHAW, B. K. Acoustic assessment of wood quality of raw forest materials - a path to increased profitability. Forest Products Journal, Madison, v. 57, n. 5, p. 614, 2007. 